\title{
DISK-TYPE INPUT COUPLER WITH A HOM DAMPER FOR THE PLS STORAGE RING CAVITY
}

\author{
J. S. Yang, I. S. Park, and M. Kwon \\ PAL/POSTECH, San-31 Hyoja-Dong, Pohang 790-784, Korea
}

\section{Abstract}

A disk-type input coupler for the Pohang Light Source (PLS) storage ring cavity was fabricated and tested up to $60 \mathrm{~kW}$. A coaxial adapter is required for this system which should have an exit for the loop coupling tubes. Since this input coupler can extract HOMs better than the cylinder-type one out of the cavity, a specially designed HOM absorber was implemented in the design of the adapter. A characteristic of the system is simulated by the HFSS code. The result of the simulation and a preliminary measurement will be presented.

\section{INTRODUCTION}

For PLS RF cavities loop couplers with cylindrical ceramic window have been used. These couplers have one drawback; the output port, WR-1500 waveguide does not match to the transmission line, which is a coaxial type. Therefore interface requires a coax-towaveguide transition which wastes small amount of RF power and makes whole system little bit complicated to handle. This motivated us to design a new disk-type coupler with coaxial port out[1]. A 3D MAFIA modeling shows good performance and low power test proved that point. It also shows a good HOM extraction characteristics which led us to develop a outside HOM absorber. A high power test and the design work for the filter of the HOM absorber will be covered in the following sections.

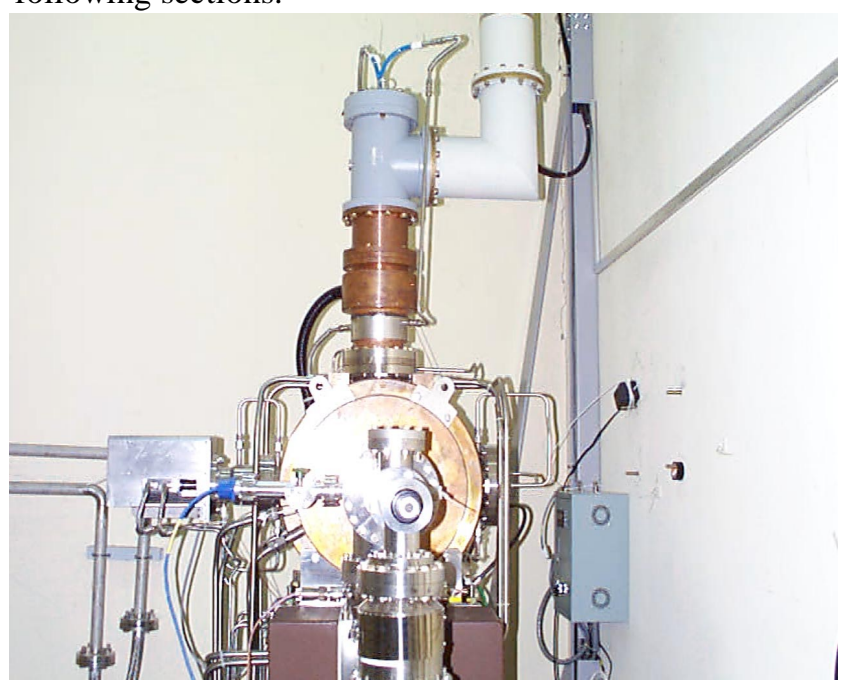

Figure 1. Prototype input coupler installed for the high power test.

\section{HIGH POWER TEST OF THE PROTOTYPE INPUT COUPLER}

The high power test stand (HPTS) in PLS consists of a $60 \mathrm{~kW}$ cw klystron amplifier, a circulator, low level control system and a spare cavity. Inside cavity the ceramic window region is monitored by a CCD camera and the reflected power, vacuum pressure and the arc signal are connected to the interlock circuit to trip off the input rf power when signal exceed the preset limits. All data for the forward and reflected power, vacuum pressure, temperatures on various points along with monitoring signal of the klystron amplifier are taken, stored and processed for display. A PC with a GPIB interface and HP DAQ modules control and monitor the whole system.

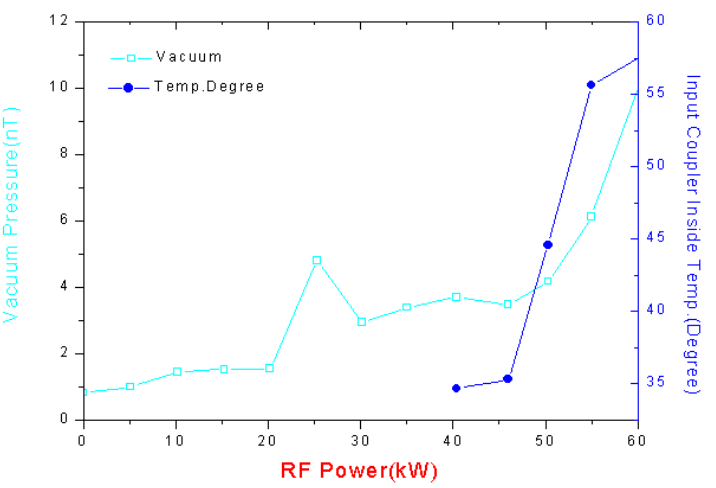

Figure 2. Changes in vacuum pressure and the temperature of the inside wall of the inner conductor during the aging of the prototype input coupler.

The high power test went through as normal. In low power region multipactoring occurred often but decreased as aging process went on. The reflected power was kept very low proving good VSWR as expected in the previous low power test. Above $40 \mathrm{~kW}$ of input power occasional arcs appeared followed by a vacuum pressure burst to $0.01 \mathrm{mT}$ range. The number of occurrence decreased substantially in two days of operations. This means that the arc may not be caused by a structural problems. A qualitative improvement of the surface treatment may be required.

A thermocouple attached to the inner wall of the center conductor shows a rapid increase of the temperature above $50 \mathrm{~kW}$ of power applied whereas the vacuum 
pressure gradually increased to a nano-Torr level (Figure 2).

\section{HOM DAMPER}

During the low power measurements, it was observed that in the prototype coupler more HOMs was coupled out compared to the previously used disk-type coupler. This is shown in Fig. 3. The spectra were measured from a loop probe placed inside cavity. For the prototype coupler less modes are visible, meaning that more HOMs are coupled outside through the disk window. To absorbed these HOMs not to turn back to the cavity, an absorber should be provided. The quarter wavelength reflection plate of the T-adapter is the best place to use if a proper way is found to filter out HOMs only. The fundamental mode should be reflected fully at this plate for minimum power loss.

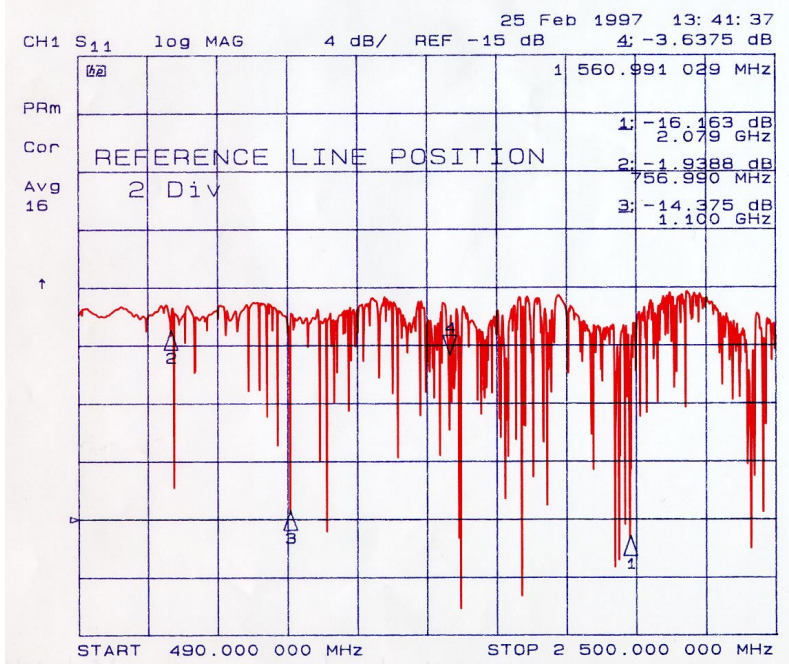

Figure 3(a). HOM spectrum for cylindrical-type window coupler.

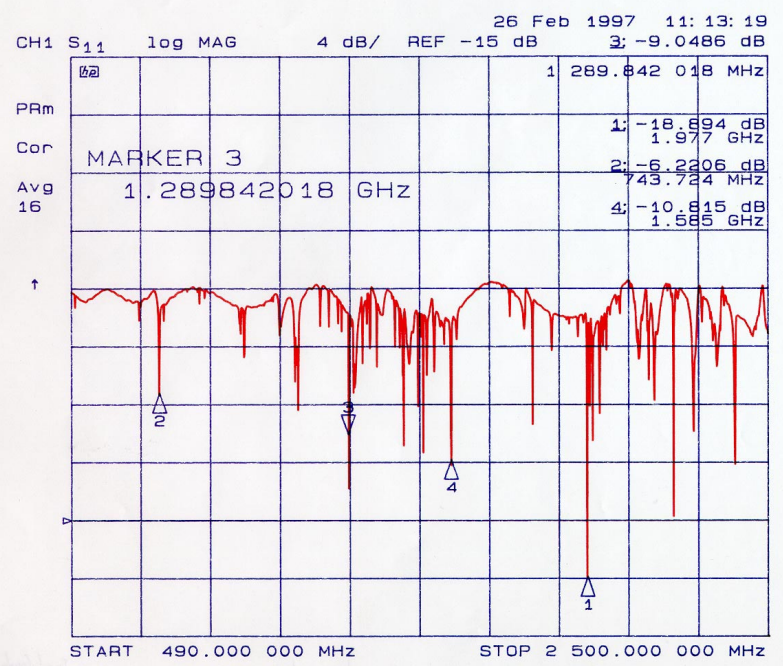

Figure 3(b). HOM spectrum for disk-type window coupler.

In the preliminary study, a simple stub-type filter was investigated. The rejection characteristics of the single stub design was reported to show poor rejection characteristics[3]. Here multi-stub design were tested initially. Simulation with the HFSS shows filter characteristics. As in Fig. 4, the initial results show that the design still need more fundamental mode rejection ratio and less attenuation in the pass-band. Optimization and study for other type of filters such as the Chebyshevtype one[2] should be pursued.
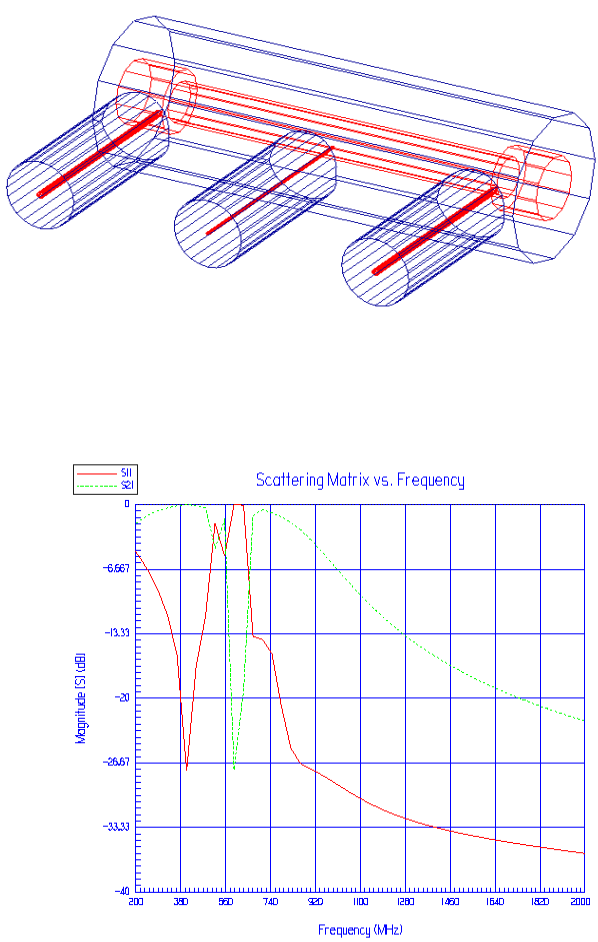

Figure 4. A model of the stub-type high pass filter simulated by the HFSS. Bottom graph shows S11 and S12 of the model filter.

For the dampers, matched load will be used. Detail design study of the damper just begins. Design, low power model and high power model development will be paralled with the T-adapter fabrication. The whole system will be tested and characterized in late this year.

\section{REFERENCES}

[1] I. S. Park, et al., 'Development of a Disk-Type Cavity Input Coupler,' in APAC98 Proceedings, Tsukuba, 1998.

[2] Y. W. Kang, et al., 'Coaxial Higher-Order Mode Damper Employing a High Pass Filter,' in PAC97 Proceedings, Vancouver, 1997.

[3] P. Matthews, et al., 'Storage Ring Cavity HigherOrder Mode Dampers for the Advanced Photon Source,' in PAC95 Proceedings, Dallas, 1995. 